\title{
CYCLIC SUBGROUP GRAPH OF A FINITE GROUP
}

\author{
J. John Arul Singh ${ }^{1}$, S. Devi ${ }^{2} \S$ \\ ${ }^{1,2}$ Department of Mathematics \\ Noorul Islam Centre for Higher Education \\ Thuckalay, 629 180, Kanyakumari, Tamilnadu, INDIA
}

\begin{abstract}
The cyclic subgroup graph $\Gamma_{z}(\mathrm{G})$ of a finite group $\mathrm{G}$ is a graph in which the cyclic subgroups are vertices and two distinct subgroups are adjacent if one of them is a subset of the other. In this paper few parameters in cyclic subgroup graph of a finite group such as independence number, chromatic number and matching number are studied. Also the independence number of power graph and cyclic subgroup graph of a finite group are found to be same.
\end{abstract}

AMS Subject Classification: $05 \mathrm{C}$

Key Words: cyclic subgroup graph, power graph, independence

\section{Introduction}

Algebraic graph theory has developed in various dimensions in the past two decades. In this paper, we relate a group with a graph by its subgroup structure.

By a graph $\Gamma=(V, E)$, we mean a connected, finite, undirected graph with neither loops nor multiple edges. For notations and terminology, refer $[1,2]$. The power graph $\mathscr{G}(G)$ of a finite group $G$ is a graph in which the group elements are the vertices and two distinct elements $x$ and $y$ are adjacent if one of them

Received: $\quad$ August 28, 2016

Revised: $\quad$ November 8, 2016

Published: December 16, 2016

${ }^{\S}$ Correspondence author
(C) 2016 Academic Publications, Ltd. url: www.acadpubl.eu 
is a power of the other. Power graph is studied in detail in [4]. The cyclic subgroup graph $\Gamma_{z}(G)$ is a graph in which the cyclic subgroups are vertices and two distinct subgroups are adjacent if one of them is a subset of the other. In this paper few parameters in cyclic subgroup graph of a finite group such as independence number, chromatic number and matching number are studied. Also the independence number of a power graph and the independence number of cyclic subgroup graph are found to be same. Let $X=\left\{x_{1}, x_{2}, \ldots, x_{n}\right\}$ be a finite set and let $\mathscr{E}=\left(E_{i} / 1 \leq i \leq m\right)$ be a family of nonempty subsets of $X$ satisfying $\bigcup_{i=1}^{m} E_{i}=X$. Then $H=(X, \mathscr{E})$ is said to be a hypergraph. We need the following results.

Definition 1. [3] Let $H=(X, \mathscr{E})$ be a hypergraph. A set $D \subset X$ is called a hyperdominating set if for each $y \in X-D$ there exist some edge $E$ containing $y$ with $|E| \geq 2$ such that $E-y \subset D$. The hyperdomination number $\gamma_{h}(H)$ is the minimum cardinality of all hyperdominating sets.

Definition 2. [3] Let $G$ be an abelian group. Then $G$ can be represented as $G \cong Z_{p_{1} n_{1}} \otimes \ldots \otimes Z_{p_{k}{ }^{n_{k}}}$ where $\otimes$ represents direct product of groups and $p_{i}^{\prime} s$ are primes. $G_{D}$ denotes a graph with vertex set

$$
V\left(G_{D}\right)=Z_{\left(n_{1}+1\right)} \times \ldots \times Z_{\left(n_{k}+1\right)},
$$

where $\times$ represents cartesian product. Two tuples $\left(x_{1}, \ldots, x_{k}\right)$ and $\left(y_{1}, \ldots, y_{k}\right)$ in $V\left(G_{D}\right)$ are adjacent if and only if either $x_{i} \geq y_{i}$ for all $i$ or $x_{i} \leq y_{i}$ for all $i$.

Lemma 3. [3] For any abelian group

$$
G \cong Z_{p_{1} n_{1}} \otimes \ldots \otimes Z_{p_{k} n_{k}},
$$

$p_{i}^{\prime} s$ are primes, the sets $S_{l}=\left\{\left(x_{1}, x_{2}, \ldots, x_{k}\right) \in Z_{\left(n_{1}+1\right)} \times \ldots \times Z_{\left(n_{k}+1\right)}: \sum x_{i}=\right.$ $l\}$ for $0 \leq l \leq \sum n_{i}$ are independent in $G_{D}$.

Theorem 4. [3] For any group $G$ of order $n$ with

$$
\begin{aligned}
& G \cong Z_{p_{1}{ }^{n_{1}}} \otimes \ldots \otimes Z_{p_{k} n_{k}},(k \geq 2), \\
& \gamma_{h}(G) \leq n-\sum_{i_{1}=0}^{\min \left\{n_{1}, n_{2}\right\}} \sum_{i_{2}=0}^{\min \left\{n_{1}+n_{2}-2 i_{1}, n_{3}\right\}} \cdots \\
& \min \left\{\sum_{j=1}^{k-2} n_{j}-2 \sum_{j=1}^{k-3} i_{j}, n_{k-1}\right\} \\
& \sum_{i_{k-2}=0} \\
& \min \left\{\sum_{j=1}^{k-1} n_{j}-2 \sum_{j=1}^{k-2} i_{j}+1, n_{k}+1\right\} .
\end{aligned}
$$

Moreover, equality holds whenever $G$ is cyclic. 
Theorem 5. [3] For any group $G$ of order $n, \gamma_{h}(G)=n-\beta_{o}(\mathscr{G}(G))$ where $\mathscr{G}(G)$ is the undirected power graph of $G$.

\section{Main Results}

Definition 6. The cyclic subgroup graph $\Gamma_{z}(G)$ of a finite group $G$ is a simple undirected graph in which the cyclic subgroups of $G$ are the vertices and two distinct subgroups are adjacent if one of them is a subset of the other.

Theorem 7. For any finite group $G$, the independence number of the cyclic subgroup graph of $G$ is same as that of the independence number of the power graph of $G$. That is $\beta_{o}\left(\Gamma_{z}(G)\right)=\beta_{o}(\mathscr{G}(G))$

Proof. Let $\beta_{o}(\mathscr{G}(G))=s$ and $A=\left\{x_{1}, x_{2}, x_{3}, \ldots x_{s}\right\}$ be a maximum independent set of $\mathscr{G}(G)$ having $s$ vertices. Take $x_{i}, x_{j} \in A$ and $i \neq j$, then $x_{i}$ and $x_{j}$ are not adjacent. Therefore $x_{i} \neq x_{j}^{k}$ and $x_{j} \neq x_{i}^{k}$ for all positive integer $k$. From this $x_{i} \notin\left\langle x_{j}\right\rangle$ and $x_{j} \notin\left\langle x_{i}\right\rangle$. So that $\left\langle x_{i}\right\rangle \nsubseteq\left\langle x_{j}\right\rangle$ and $\left\langle x_{j}\right\rangle \nsubseteq\left\langle x_{i}\right\rangle$. Therefore $\left\{\left\langle x_{1}\right\rangle,\left\langle x_{2}\right\rangle, \ldots\left\langle x_{s}\right\rangle\right\}$ is an independent set of $\Gamma_{z}(G)$. So that any maximum independent set of $\Gamma_{z}(G)$ has atleast $s$ vertices. Therefore $\beta_{o}\left(\Gamma_{z}(G)\right) \geq \beta_{o}(\mathscr{G}(G))$.

For the other inequality take $\beta_{o}\left(\Gamma_{z}(G)\right)=s$.

Let $B=\left\{\left\langle y_{1}\right\rangle,\left\langle y_{2}\right\rangle,\left\langle y_{3}\right\rangle, \ldots .\left\langle y_{s}\right\rangle\right\}$ be a maximum independent set of $\Gamma_{z}(G)$ having $s$ vertices. Let $\left\langle y_{i}\right\rangle,\left\langle y_{j}\right\rangle \in B$ and $i \neq j$, then $\left\langle y_{i}\right\rangle$ and $\left\langle y_{j}\right\rangle$ are not adjacent in $\Gamma_{z}(G)$. Therefore $\left\langle y_{i}\right\rangle \nsubseteq\left\langle y_{j}\right\rangle$ and $\left\langle y_{j}\right\rangle \nsubseteq\left\langle y_{i}\right\rangle$. From this $y_{i} \notin\left\langle y_{j}\right\rangle$ and $y_{j} \notin\left\langle y_{i}\right\rangle$. Hence $y_{i} \neq y_{j}^{k}$ and $y_{j} \neq y_{i}^{k}$ for all $k$. It follows that $y_{i}$ and $y_{j}$ are not adjacent to each other in $\mathscr{G}(G)$. Since $y_{i}$ and $y_{j}$ are taken arbitrary, $\left\{y_{1}, y_{2}, y_{3}, . . y_{s}\right\}$ is an independent set in $\mathscr{G}(G)$. Therefore the maximum independent set of $\mathscr{G}(G)$ has atleast $s$ vertices. Therefore $\beta_{o}\left(\Gamma_{z}(G)\right) \leq$ $\beta_{o}(\mathscr{G}(G))$.

Theorem 8. The independence number of the cyclic subgroup graph of any finite cyclic group $Z_{n}$,

$$
\begin{gathered}
\beta_{o}\left(\Gamma_{z}\left(Z_{n}\right)\right)=\sum_{i_{1}=0}^{\min \left\{n_{1}, n_{2}\right\}} \sum_{i_{2}=0}^{\min \left\{n_{1}+n_{2}-2 i_{1}, n_{3}\right\}} \cdots \\
\sum_{i_{k-2}=0}^{\min \left\{\sum_{j=1}^{k-2} n_{j}-2 \sum_{j=1}^{k-3} i_{j}, n_{k-1}\right\}} \min \left\{\sum_{j=1}^{k-1} n_{j}-2 \sum_{j=1}^{k-2} i_{j}+1, n_{k}+1\right\} .
\end{gathered}
$$


Proof. From theorem 4 and theorem 5 it follows that the independence number of the power graph of $Z_{n}$,

$$
\begin{gathered}
\beta_{o}\left(\mathscr{G}\left(Z_{n}\right)\right)=\sum_{i_{1}=0}^{\min \left\{n_{1}, n_{2}\right\}} \sum_{i_{2}=0}^{\min \left\{n_{1}+n_{2}-2 i_{1}, n_{3}\right\}} \cdots \\
\sum_{i_{k-2}=0}^{\min \left\{\sum_{j=1}^{k-2} n_{j}-2 \sum_{j=1}^{k-3} i_{j}, n_{k-1}\right\}} \min \left\{\sum_{j=1}^{k-1} n_{j}-2 \sum_{j=1}^{k-2} i_{j}+1, n_{k}+1\right\} .
\end{gathered}
$$

Now applying theorem 7 result follows.

Theorem 9. For any cyclic group $Z_{n} \cong Z_{p_{1}{ }^{n_{1}}} \otimes \ldots \otimes Z_{p_{k}{ }^{n_{k}}}, p_{i}^{\prime} s$ are distinct primes, $\Gamma_{z}\left(Z_{n}\right)$ is isomorphic to $Z_{n D}$.

Proof. In $Z_{n}$ every subgroup is cyclic and each subgroup is of the form

$$
<p_{1}^{r_{1}}>\otimes<p_{2}^{r_{2}}>\otimes \ldots \otimes<p_{k}^{r_{k}}>
$$

where $0 \leq r_{i} \leq n_{i}$. Define $f: V\left(\Gamma_{z}\left(Z_{n}\right)\right) \longrightarrow V\left(Z_{n D}\right)$ by

$$
f\left(<p_{1}^{r_{1}}>\otimes<p_{2}^{r_{2}}>\otimes \ldots \otimes<p_{k}^{r_{k}}>\right)=\left(r_{1}, r_{2}, \ldots, r_{k}\right) .
$$

Then $f$ is a bijection. Also

$$
<p_{1}^{r_{1}}>\otimes<p_{2}^{r_{2}}>\otimes \ldots \otimes<p_{k}^{r_{k}}>\subseteq<p_{1}^{s_{1}}>\otimes<p_{2}^{s_{2}}>\otimes \ldots \otimes<p_{k}^{s_{k}}>
$$

holds if and only if $r_{i} \leq s_{i} \forall i$ holds. So that $\Gamma_{z}\left(Z_{n}\right)$ is isomorphic to $Z_{n D}$.

Theorem 10. For any cyclic group $Z_{n} \cong Z_{p_{1}{ }^{n_{1}}} \otimes \ldots \otimes Z_{p_{k}{ }^{n_{k}}}, p_{i}^{\prime} s$ are distinct primes, the chromatic number of the cyclic subgroup graph of $Z_{n}$, $\chi\left(\Gamma_{z}\left(Z_{n}\right)\right)=n_{1}+n_{2}+\ldots+n_{k}+1$.

Proof. From theorem 10 its enough to find the chromatic number of $Z_{n D}$. Consider the sets $S_{l}=\left\{\left(x_{1}, x_{2}, \ldots, x_{k}\right) \in Z_{\left(n_{1}+1\right)} \times \ldots \times Z_{\left(n_{k}+1\right)}: \sum_{i=1}^{k} x_{i}=l\right\}$ for $0 \leq l \leq \sum n_{i}$. By Theorem 3 each of these sets $S_{l}$ are independent. So that $\left\{S_{0}, S_{1}, S_{2}, \ldots, S_{n_{1}+n_{2}+\ldots+n_{k}}\right\}$ forms a chromatic partition of $\Gamma_{z}\left(Z_{n}\right)$. Hence $\chi\left(\Gamma_{z}\left(Z_{n}\right)\right) \leq n_{1}+n_{2}+\ldots+n_{k}+1$.

Now consider the set $K=\left\{\left(n_{1}, n_{2}, \ldots, n_{i-1}, t_{i}, 0,0, \ldots, 0\right): 0 \leq t_{i} \leq n_{i}, 1 \leq\right.$ $i \leq k\}$. Any two vertices in $K$ are adjacent. So that $K$ induces a complete subgraph with $|K|=n_{1}+n_{2}+\ldots+n_{k}+1$. Hence $\chi\left(\Gamma_{z}\left(Z_{n}\right)\right) \geq n_{1}+n_{2}+\ldots+$ $n_{k}+1$ which completes the proof. 
Theorem 11. For any cyclic group $Z_{n} \cong Z_{p_{1} n_{1}} \otimes \ldots \otimes Z_{p_{k}{ }^{n_{k}}}, p_{i}^{\prime} s$ are distinct primes, the cyclic subgroup graph $\Gamma_{z}\left(Z_{n}\right)$ is Hamiltonian.

Proof. By Theorem 9 it's enough to prove $Z_{n D}$ is Hamiltonian. The vertex $(0,0,0, \ldots, 0) \in V\left(Z_{n D}\right)=Z_{\left(n_{1}+1\right)} \times \ldots \times Z_{\left(n_{k}+1\right)}$ denoted by origin is adjacent to all other vertices. So it's enough to prove there is a Hamiltonian path in $Z_{n D}$ starting from the origin. We prove this by induction on $k$. Suppose $k=1$. Then $Z_{n} \cong Z_{p_{1} n_{1}}$. Now $P=\left(0,1,2, \ldots, n_{1}\right)$ is a Hamiltonian path starting from origin 0 . So the result is true for $k=1$. Assume the result is true for $k-1$. To prove for $k$ assume $Z_{n} \cong Z_{p_{1} n_{1}} \otimes \ldots \otimes Z_{p_{k}{ }^{n_{k}}}, p_{i}^{\prime} s$ are distinct primes. Take $m=p_{1}^{n_{1}} \otimes \ldots \otimes p_{k-1}{ }^{n_{k-1}}$. Then $Z_{m} \cong Z_{p_{1} n_{1}} \otimes \ldots \otimes Z_{p_{k-1} n_{k-1}}$. By our assumption $Z_{m D}$ has a Hamiltonian path starting from origin say $P=(0,0, \ldots, 0)=$ $\left(x_{11}, x_{12}, \ldots, x_{1(k-1)}\right),\left(x_{21}, x_{22}, \ldots, x_{2(k-1)}\right), \ldots,\left(x_{t 1}, x_{t 2}, \ldots, x_{t(k-1)}\right)$ where $t=$ $\left(n_{1}+1\right)\left(n_{2}+1\right) \ldots\left(n_{k-1}+1\right)$.

Consider the corresponding paths

$$
\begin{array}{r}
P_{i}=\left((0,0, \ldots, 0, i)=\left(x_{11}, x_{12}, \ldots, x_{1(k-1)}, i\right),\left(x_{21}, x_{22},\right.\right. \\
\left.\left.\ldots, x_{2(k-1)}, i\right), \ldots,\left(x_{t 1}, x_{t 2}, \ldots, x_{t(k-1)}, i\right)\right)
\end{array}
$$

for $0 \leq i \leq n_{k}$. Let the reverse path be denoted by

$$
\begin{array}{r}
P_{i}^{-1}=\left(\left(x_{t 1}, x_{t 2}, \ldots, x_{t(k-1)}, i\right), \ldots,\left(x_{21}, x_{22}, \ldots, x_{2(k-1)}, i\right),(0,0, \ldots, 0, i)\right. \\
\left.=\left(x_{11}, x_{12}, \ldots, x_{1(k-1)}, i\right)\right) .
\end{array}
$$

Now consider the path $P^{\prime}=P_{0} P_{1}^{-1} P_{2} P_{3}^{-1} P_{4} \ldots P^{*}$ taking the $P_{i}$ and their inverse paths alternatively in the sense the path starts from vertices of $P_{0}$ followed by vertices of $P_{1}^{-1}$ followed by the vertices of $P_{2}$ and so on until the last path $P^{*}$ which may be either $P_{n_{k}}$ or $P_{n_{k}}^{-1}$ depending on whether $n_{k}$ is even or odd. $P^{\prime}$ is the required Hamiltonian path in $Z_{n D}$ starting from the origin.

Theorem 12. For any cyclic group $Z_{n} \cong Z_{p_{1} n_{1}} \otimes \ldots \otimes Z_{p_{k} n_{k}}, p_{i}^{\prime} s$ are distinct primes, the matching number of $\Gamma_{z}\left(Z_{n}\right)$ is $\left\lfloor\frac{n}{2}\right\rfloor$.

Proof. By theorem 11 there is Hamiltonian cycle for $\Gamma_{z}\left(Z_{n}\right)$ say $C_{n}=\left(v_{1}, v_{2}, v_{3}, \ldots, v_{n}, v_{1}\right)$. When $n$ is even taking the alternative edges $\left\{v_{1} v_{2}, v_{3} v_{4}, v_{5} v_{6}, \ldots, v_{n-1} v_{n}\right\}$ a perfect matching is obtained. When $n$ is odd $\left\{v_{1} v_{2}, v_{3} v_{4}, v_{5} v_{6}, \ldots, v_{n-2} v_{n-1}\right\}$ forms a maximum matching with $\frac{n-1}{2}$ vertices. Hence $\Gamma_{z}\left(Z_{n}\right)$ has matching number $\left\lfloor\frac{n}{2}\right\rfloor$. 


\section{References}

[1] Frank Hahary, Graph Theory, Addison-Wesley Publishing Company Reading, Massachusetts Menlo Park, California London Don Mills, 1969.

[2] I.N. Herstein, Topics in Algebra, John Wiley and Sons, New York-Chichester-BrisbaneToronto-Singapore, 1975.

[3] J. John Arul Singh and R. Kala, Hyperdomination in groups, Ars Combinatoria, 125 (2016), 97-108.

[4] Peter J. Cameron, Shamik Ghosh, The power graph of a finite group, Discrete Mathematics, 311, Iss 13 (2011), 1220-1222. 